\title{
Pancreatoduodenectomía laparoscópica
}

\author{
Laparoscopic pancreatoduodenectoy
}

\author{
Gustavo A. Guerrero-Martínez ${ }^{1 *}$, Roberto Estrada-Gómez², Armando Basilio-Roque ${ }^{3}$, \\ Rafael Viveros-Luna ${ }^{3}$, César Lorenzo-Yacamix y Pablo A. Dávila-Esparza ${ }^{4}$ \\ 'Servicio de Cirugía General y Laparoscopía, Hospital Regional de Alta Especialidad, Instituto de Seguridad y Servicios Sociales de los Trabajadores \\ del Estado (ISSSTE); 2 Servicio de Cirugía General, Laparoscopía y Cirugía de Obesidad, Hospital Angeles Puebla; ${ }^{3}$ Servicio de Oncología \\ Quirúrgica, Hospital Regional de Alta Especialidad, ISSSTE; ${ }^{4}$ Servicio de Cirugía General, Hospital Regional de Alta Especialidad, ISSSTE. Puebla, \\ México
}

\section{Resumen}

Antecedentes: La pancreatoduodenectomía o cirugía de Whipple es el procedimiento de elección en el tratamiento de las enfermedades periampulares. A través de los años se ha realizado de manera abierta, y actualmente, gracias a la mejora de los recursos humanos y tecnológicos, se realiza este procedimiento por mínima invasión con buenos resultados. Objetivo: Presentar la experiencia inicial en la pancreatoduodenectomía totalmente laparoscópica en el Hospital de Alta Especialidad del Instituto de Seguridad y Servicios Sociales de los Trabajadores del Estado (ISSSTE) de Puebla, dando a conocer la segunda serie de casos reportada en México. Método: De julio de 2014 a julio de 2018 se han realizado ocho pancreatoduodenectomías totalmente laparoscópicas, siete en el hospital de alta especialidad ISSSTE Puebla y una en un hospital privado. Resultados: En todos los pacientes intervenidos los resultados son favorables, sin mortalidad transoperatoria y sin presentar complicaciones graves ni reintervención. Conclusión: La pancreatoduodenectomía laparoscópica es un procedimiento de alta complejidad que, teniendo una adecuada selección de pacientes y un manejo multidisciplinario, se puede llevar a cabo con excelentes resultados.

Palabras Clave: Pancreatoduodenectomía laparoscópica. Procedimiento de Whipple. Cirugía pancreática.

\begin{abstract}
Background: Pancreatoduodenectomy or Whipple's operation, is the elective surgical procedure to treat different periampullary diseases. Through the years this surgery has been doing open, but in the lasts decades thanks to the improve technology and trained surgeons, today is feasible doing it laparoscopic with good results. Objective: To present the initial experience and results in totally laparoscopic pancreatoduodenectomy in Hospital Regional ISSSTE Puebla, reporting the second number of cases in México. Method: Since July 2014-July 2018, 8 patients has been operated by totally laparoscopic pancreatoduodenectomy, 7 in Hospital Regional ISSSTE Puebla and 1 in a private Hospital. Results: Evaluating all the patients, not one had mortality during operation, no morbidity or immediate reoperation, so the results are favourable. Conclusion: Laparoscopic pancreatoduodenectomy is a very complex procedure but feasible, and good results depends on various factors, like the appropriate patient selection.
\end{abstract}

Key Words: Laparoscopic pancreatoduodenectomy. Whipple procedure. Pancreatic surgery.

\section{Correspondencia:}

*Gustavo A. Guerrero-Martínez

Privada de las Ramblas, 4

Col. Desarrollo Atlixcáyotl

Fecha de recepción: 18-01-2018

C.P. 72197, Puebla, México

Fecha der aceptación: 09-12-2019

E-mail: dr.guerrerom@yahoo.com.mx

DOI: $10.24875 / C I R U .19000093$

Cir Cir. 2020;88(3):263-268

Contents available at PubMed www.cirugiaycirujanos.com

0009-7411/@ 2019 Academia Mexicana de Cirugía. Publicado por Permanyer. Este es un artículo open access bajo la licencia CC BY-NC-ND (http://creativecommons.org/licenses/by-nc-nd/4.0/). 


\section{Introducción}

Los tumores periampulares se caracterizan por ser un grupo heterogéneo de neoplasias de diferente estirpe histológica y son todos aquellos tumores confinados a esta región anatómica, es decir, tumores de cabeza de páncreas (el más frecuente), ampolla de Váter, vía biliar principal distal y duodeno'. Por su ubicación anatómica comprometen el área de confluencia biliopancreática y producen un cuadro similar y ominoso; además, involucran numerosas estructuras viscerales y vasculares para su resección.

La pancreatoduodenectomía es el procedimiento quirúrgico de elección para el tratamiento de los tumores malignos periampulares, las neoplasias pancreáticas quísticas localizadas en la cabeza del páncreas $\mathrm{y}$, en algunas ocasiones, pacientes con trauma abdominal. Este procedimiento se conoce también como procedimiento de Whipple, descrito por primera vez en 1934 por Allan Whipple ${ }^{2}$, y se ha realizado en numerosos hospitales de todo el mundo. Sin embargo, este tipo de cirugía realizada por mínima invasión (laparoscopía) se lleva a cabo cada vez con mayor frecuencia. En México, en muy pocos centros se ha realizado por esta vía con buenos resultados.

En los Estados Unidos se pronosticó que para el año 2015 serían diagnosticadas más de 48,000 personas con cáncer de páncreas ${ }^{3}$, y que poco más del $80 \%$ morirían a causa de esta enfermedad. En México, en el último estudio realizado, se reporta un incremento en el número de casos y una mortalidad mayor del $90 \%{ }^{4}$; el cáncer de páncreas tiene una alta morbimortalidad y ocupa el cuarto lugar en el mundo y el octavo en nuestro país como causa de muerte por enfermedad relacionada con cáncer ${ }^{1}$.

Como ya se mencionó, la cirugía es la única opción potencialmente curativa para este tipo de enfermedades, y en el caso del cáncer de cabeza de páncreas solo el $15-20 \%$ de los pacientes son candidatos a resección quirúrgica; aun así, la sobrevida a 5 años posterior a la pancreatoduodenectomía con resección R0 y R1 es del $25-30 \%$ y del 10\%, respectivamente 2 .

El adenocarcinoma de la ampolla de Váter representa el $10-36 \%$ del total de las neoplasias malignas periampulares resecables, con un $76-78 \%$ de posibilidad de resecabilidad, a diferencia del $22 \%$ de los pacientes con tumores cefálicos de páncreas. La sobrevida a 5 años en los pacientes posoperados de pancreatoduodenectomía por ampuloma es del $32-58 \%{ }^{1,4}$.
Tabla 1. Procedimientos quirúrgicos realizados por laparoscopía

\begin{tabular}{lc}
\hline \multicolumn{2}{c}{ Cirugía realizada } \\
\hline Coledocoduodenostomía & 12 \\
Hepaticoyeyunostomía en Y de Roux & 10 \\
Pancreatectomía distal con preservación esplénica & 1 \\
Pancreatoduodenectomía & 8 \\
\hline
\end{tabular}

\section{Método}

En el Hospital Regional de Alta Especialidad ISSSTE Puebla, de octubre de 2009 a noviembre de 2017 se tienen registradas 17 pancreatoduodenectomías abiertas por diversas enfermedades, principalmente malignas y en su mayoría adenocarcinoma de cabeza de páncreas (70\%). A partir del año de 2014, en el hospital se empezó a realizar el procedimiento por vía totalmente laparoscópica. Todos los pacientes elegidos para este procedimiento fueron evaluados por los servicios de cirugía general y laparoscopía, cirugía hepatopancreatobiliar y cirugía oncológica tomando en cuenta los diferentes criterios para proponer la resección quirúrgica. Los pacientes que no cumplían las condiciones para ofrecer tratamiento curativo fueron manejados con tratamiento paliativo o biopsia y terapia neoadyuvante. Para este estudio se incluyeron únicamente los pacientes que fueron sometidos a resección quirúrgica de inicio.

De julio de 2014 a julio de 2018, ocho pacientes fueron operados de pancreatoduodenectomía totalmente laparoscópica por los mismos cirujano y equipo quirúrgico. El rango de edad de los pacientes fue de 36-79 años. Las patologías por las que fueron intervenidos fueron adenocarcinoma de ámpula de Vater (tres pacientes) y adenocarcinoma de cabeza de páncreas (cinco pacientes) (Tabla 1). El hospital cuenta con todos los recursos humanos y tecnológicos, tales como endoscopía, ultrasonido, tomografía, resonancia magnética y ultrasonido endoscópico; y se involucran los servicios de cirugía endoscópica, cirugía hepatopancreatobiliar, unidad de cuidados intensivos, anatomía patológica y oncología quirúrgica y médica, para la adecuada evaluación, la selección, el manejo posoperatorio y el seguimiento de pacientes candidatos a este tipo de procedimiento, con el fin de ofrecer mejores resultados, siempre apegados a las guías nacionales e internacionales (Figs. 1 a 3).

\section{Técnica quirúrgica}

Los pacientes se colocaron en posición de Trendelemburg, con ambos brazos pegados al cuerpo, el cirujano 


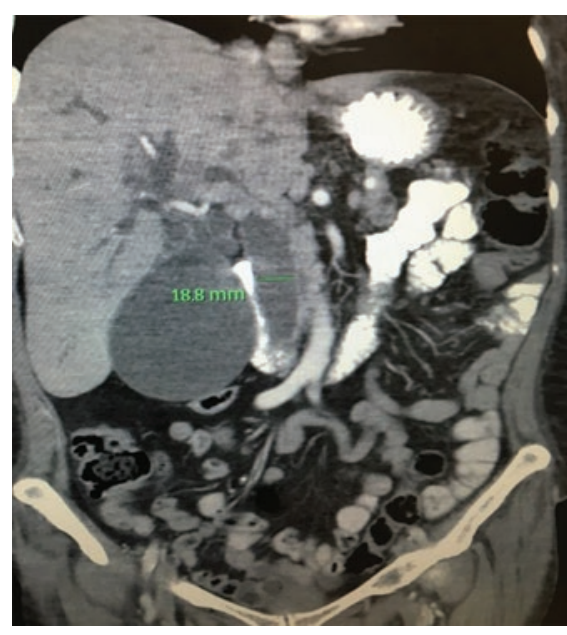

Figura 1. Tomografía con doble contraste en la que se observa dilatación de la vía biliar intrahepática y extrahepática secundaria a un tumor maligno de ampolla de Váter.

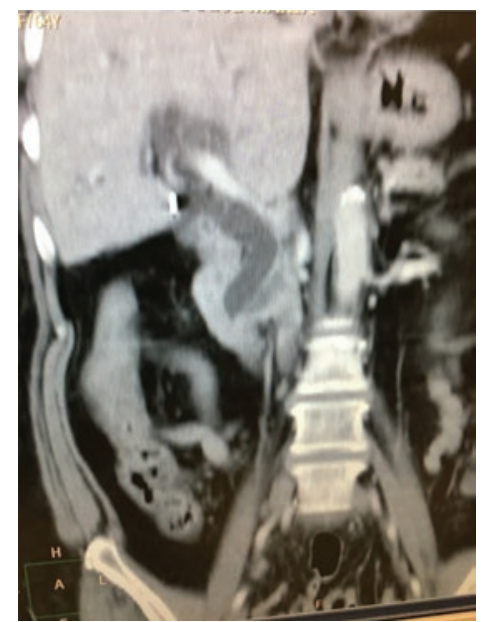

Figura 2. Tomografía con doble contraste de paciente con tumoración de cabeza de páncreas.

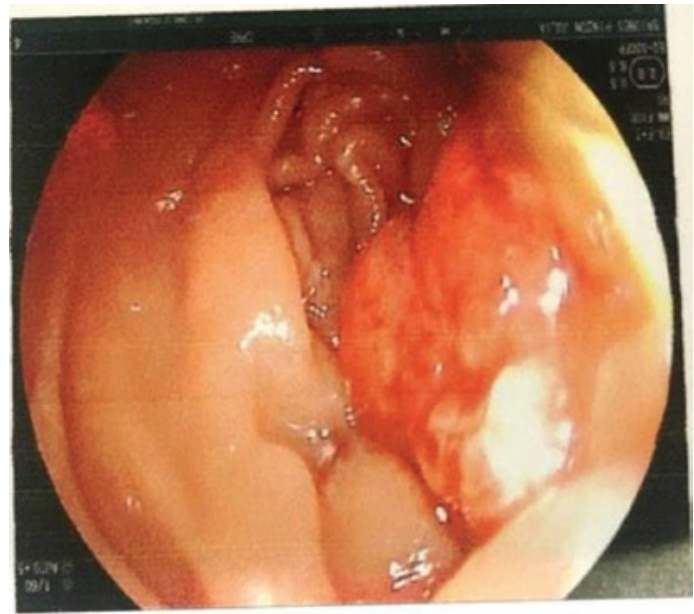

Figura 3. Tumor de ampolla de Váter visto por endoscopia.

entre las piernas y los asistentes a los lados. Se realizó neumoperitoneo a $12 \mathrm{mmHg}$ con técnica de Hasson y bajo visión directa se colocaron cuatro puertos de trabajo

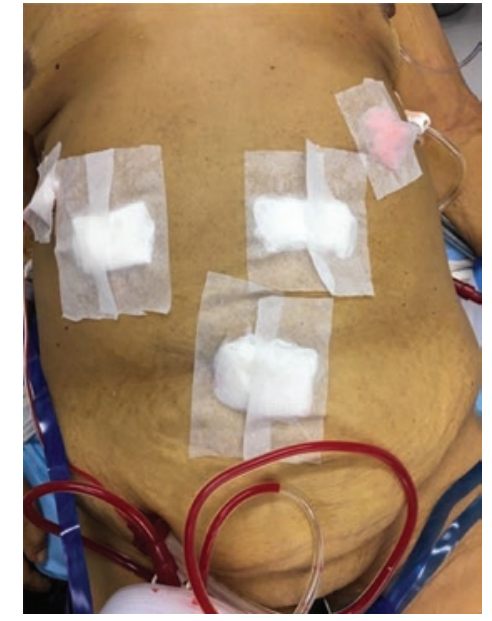

Figura 4. Posición de los trocares.

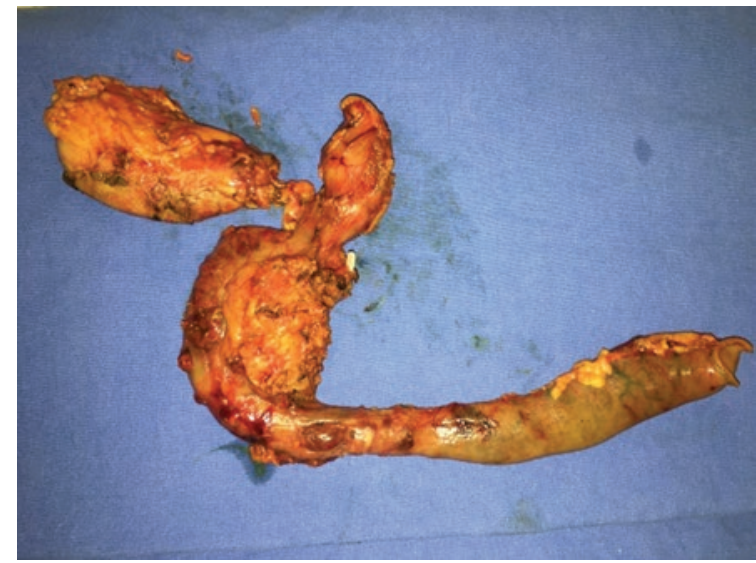

Figura 5. Producto de pancreatoduodenectomía laparoscópica.

(Fig. 4). Se inicia con revisión de la cavidad en busca de ascitis o implantes tumorales, maniobra de Kocher extendida hasta la exposición de la vena cava y el borde lateral derecho de la aorta, apertura de epiplón gastrocólico y control vascular de gastroepiploicos con bisturí armónico, y exposición de la glándula pancreática. Se localizan los vasos mesentéricos superiores, se realiza ventana retropancreática a nivel del cuello del páncreas entre la glándula y la vena porta, y suspensión del páncreas con drenaje tipo Penrose. A continuación, sección del estómago en la porción del antro con engrapadora Echelon Flex ${ }^{\circledast}$ y cartuchos azules, apertura del ligamento hepatoduodenal exponiendo el pedículo hepático y localizando la arteria gastroduodenal, control vascular con Hemo-lock ${ }^{\circledR}$ y sección de la arteria, y corte de la vía biliar principal. Se prosigue con sección del páncreas con energía bipolar a nivel del cuello, sección de duodeno-yeyuno a $10 \mathrm{~cm}$ de ángulo de Treitz y liberación completa del duodeno, despegamiento de la porción cefálica del páncreas de la lámina retroportal con Enseal ${ }^{\circledR}$ y/o bisturí armónico hasta la liberación completa (Figs. 5 


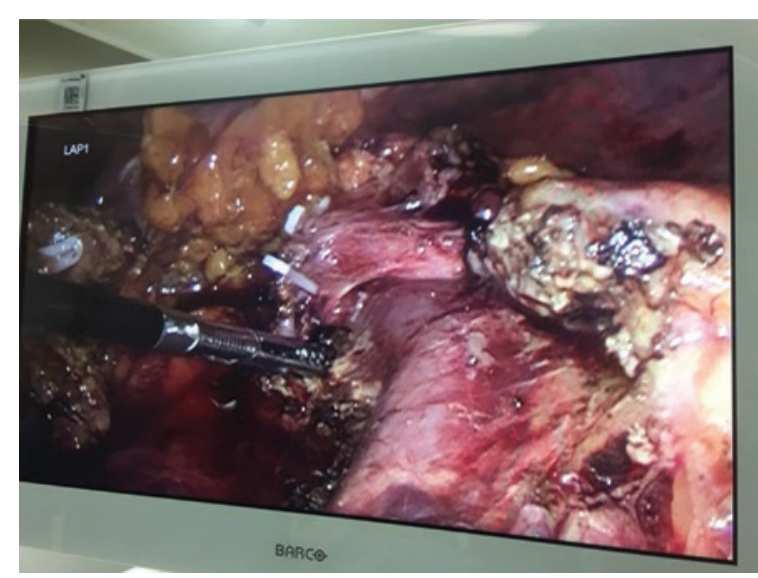

Figura 6. Lecho quirúrgico posterior a una pancreatoduodenectomía.

y 6). Se realiza reconstrucción antecólica, puntos seromusculares del yeyuno al parénquima pancreático con Prolene 2-0 línea posterior, apertura del yeyuno con energía monopolar, anastomosis ducto-mucosa Wirsung-yeyuno con Monocryl 4-0 con stent transanastomótico (en todos los casos), puntos en la línea anterior de la anastomosis seromuscular de yeyuno a páncreas con Prolene 2-0, anastomosis biliodigestiva con Monocryl 3-0 y anastomosis gastroyeyunal en la cara posterior del estómago con engrapadora Echelon Flex ${ }^{\circledR}$ de $65 \mathrm{~mm}$ cartucho azul. Se dejan dos drenajes aspirativos en la cavidad abdominal. A todos los pacientes se les inició dieta enteral a los 4 días de la cirugía, y la consulta de revisión fue a los 10 días de posoperatorio.

\section{Resultados}

De los ocho pacientes que fueron sometidos a pancreatoduodenectomía laparoscópica, cinco eran mujeres y tres eran hombres. En seis de los casos se dio aporte nutricional enteral preoperatorio. En siete pacientes la resección y la reconstrucción fueron totalmente por vía laparoscópica, sin mortalidad transopeatatoria ni complicaciones en el posoperatorio inmediato relacionados con el procedimiento quirúrgico (fístulas, colecciones, sangrado postquirúrgico, etc.). En un paciente se realizó conversión a cirugía tradicional por presentar sangrado transoperatorio que no se logró controlar por mínima invasión; no obstante, tuvo un posoperatorio favorable. Tres pacientes requirieron estancia en la unidad de cuidados intensivos y el resto continuaron el posoperatorio en piso de cirugía. Los resultados de tiempo quirúrgico, sangrado, morbilidad y estancia hospitalaria fueron favorables y muy similares a los de otros reportes nacionales e internacionales (Tablas 2 y 3 ).
Tabla 2. Distribución por edad, sexo, tipo histopatológico y días de estancia en la unidad de cuidados intensivos en los pacientes sometidos a pancreatoduodenectomía laparoscópica

\begin{tabular}{|c|c|c|c|c|}
\hline Sexo & Edad & $\begin{array}{l}\text { Estudio } \\
\text { histopatológico }\end{array}$ & $\begin{array}{c}\text { Estancia } \\
\text { hospitalaria }\end{array}$ & $\begin{array}{c}\text { Estancia } \\
\text { en UCl }\end{array}$ \\
\hline Mujer & 68 & $\begin{array}{l}\text { Adenocarcinoma de } \\
\text { ampolla de Vater }\end{array}$ & 19 días & 13 días \\
\hline Hombre & 62 & $\begin{array}{l}\text { Adenocarcinoma de } \\
\text { cabeza páncreas }\end{array}$ & 9 días & 0 \\
\hline Mujer & 57 & $\begin{array}{l}\text { Adenocarcinoma de } \\
\text { cabeza de páncreas }\end{array}$ & 10 días & 1 día \\
\hline Hombre & 36 & $\begin{array}{l}\text { Adenocarcinoma de } \\
\text { cabeza de páncreas }\end{array}$ & 9 días & 2 días \\
\hline Mujer & 64 & $\begin{array}{l}\text { Adenocarcinoma de } \\
\text { ampolla de Váter }\end{array}$ & 10 días & 0 días \\
\hline Mujer & 79 & $\begin{array}{l}\text { Adenocarcinoma de } \\
\text { cabeza de páncreas }\end{array}$ & 10 días & 0 días \\
\hline Hombre & 43 & $\begin{array}{l}\text { Adenocarcinoma de } \\
\text { cabeza de páncreas }\end{array}$ & 10 días & 0 días \\
\hline Mujer & 66 & $\begin{array}{l}\text { Adenocarcinoma de } \\
\text { ampolla de Váter }\end{array}$ & 10 días & 0 días \\
\hline
\end{tabular}

UCl: unidad de cuidados intensivos.

Tabla 3. Patologías resueltas de manera quirúrgica en el Hospital Regional de Alta Especialidad ISSSTE Puebla

\begin{tabular}{lc}
\hline Patología & Pacientes \\
\hline Coledocolitiasis & 11 \\
Quiste de colédoco & 2 \\
Síndrome de Mirizzi & 1 \\
Lesión de vía biliar & 3 \\
Ampuloma & 3 \\
Tumor sólido pseudopapilar de páncreas (cabeza y cola) & 2 \\
Adenocarcinoma de cabeza de páncreas & 5 \\
Síndrome de Lemmel & 1 \\
\hline
\end{tabular}

\section{Discusión}

La cirugía pancreática siempre se ha considerado de difícil manejo. En 1886, Nicolas Senn escribió: «La condición más favorable para una resección pancreática es cuando la enfermedad se localiza en la cola del páncreas». Fue en 1912 cuando Walter Kausch, con las aportaciones de Theodor Kocher, entre otros, llevó a cabo la primera pancreatoduodenectomía exitosa, y posteriormente, en 1935, a Allen Whipple se le atribuye la máxima aportación al tratamiento quirúrgico de los tumores periampulares, en especial el cáncer de páncreas ${ }^{4-7}$. 
Las enfermedades de las vías biliares y del páncreas son muy diversas e involucran tanto enfermedades benignas como malignas, inflamatorias y obstructivas, que en la mayoría de los casos, por la complejidad para su manejo y tratamiento, ya sea curativo o paliativo, requieren la intervención de diferentes especialistas para una resolución adecua$\mathrm{da}^{8}$. La cirugía pancreática de mínima invasión se inició en la década de 1990, principalmente en enfermedades de tipo benigno, como complicaciones de pacientes con pancreatitis agudas y crónicas ${ }^{9}$; en los casos de enfermedad maligna, únicamente para toma de biopsias, cirugía paliativa y laparoscopía estadificadora ${ }^{10-12}$.

En la actualidad se han reportado resecciones mayores por laparoscopía con resultados muy alentadores, e inclusive la pancreatectomía distal con o sin preservación esplénica se está posicionando como el procedimiento de elección para enfermedades benignas o malignas del páncreas distal13-16. En el caso específico del procedimiento de Whipple, en 1994 Gagner y Pomp describieron por primera vez la pancreatoduodenectomía laparoscópica en un paciente con pancreatitis crónica ${ }^{3}$. En México, actualmente hay reportes de dos centros donde se ha realizado este procedimiento: el Hospital General de México, que en 2015 publicó el primer caso de pancreatoduodenectomía laparoscópica, y el Hospital Manuel Gea González, con la serie más grande que se ha reportado, de seis casos.

Posterior a la primera pancreatoduodenectomía laparoscópica se han realizado diversos estudios comparando la pancreatoduodenectomía tradicional con la cirugía por laparoscopía, con resultados muy similares ${ }^{17,18}$. Después de 10 años, numerosos estudios afirman que, en términos de seguridad, costos, viabilidad y resultados oncológicos, la pancreatoduodenectomía laparoscópica tiene más ventajas que la pancreatoduodenectomía abierta ${ }^{19}$. Por otro lado, además del inicio más temprano de la vía oral, el menor sangrado, el menor dolor, la recuperación más rápida y la estancia hospitalaria más corta, destacan los beneficios que aporta la cirugía de mínima invasión. Todo ello siempre haciendo énfasis en la importancia de una adecuada y rigurosa selección del paciente, lo que permite ofrecer mejores resultados ${ }^{18,20}$.

En todos los reportes publicados, la limitante, como mencionan la mayoría de las series, es el alto grado de complejidad técnica, ya que involucra resecciones parciales o totales de dos o más órganos, disección vascular y anastomosis digestivas complejas, lo que requiere cierto número de casos (en algunos reportes, la curva de aprendizaje se establece en al menos 30 procedimientos para dominar la técnica) ${ }^{16,18}$.

Los resultados que se han obtenido han sido comparados con la cirugía tradicional y se ha reportado similares mortalidad transoperatoria y morbilidad posquirúrgica (5\% y $30-40 \%$, respectivamente) $)^{16,18}$.

\section{Conclusión}

El Hospital Regional de Alta Especialidad del ISSSTE en Puebla es un hospital regional que concentra población de todos los municipios del Estado. Además, es centro de referencia de otros estados de la República, y por lo tanto la cantidad de pacientes que se atienden anualmente es muy alta. Desde julio de 2014 hasta julio de 2018 se realizaron en el hospital diversos procedimientos, como derivaciones biliodigestivas por enfermedad benigna y maligna de vías biliares y páncreas (Tablas 2 y 3), resecciones pancreáticas parciales y ocho pancreatoduodenectomías totalmente laparoscópicas (siete en el Hospital Regional de Alta Especialidad ISSSTE Puebla y una en un hospital privado en la misma ciudad). En todos los casos se han obtenido buenos resultados en las variantes que se evaluaron, sin mortalidad transoperatoria ni reintervención en ningún paciente.

Es muy importante mencionar que hasta la fecha son solo dos los centros en México donde se han reportado series de casos de pancreatoduodenectomía y reconstrucción totalmente laparoscópica: el Hospital General Manuel Gea González y el Hospital Regional de Alta Especialidad del ISSSTE Puebla, afortunadamente con excelentes resultados. Ello demuestra que es factible realizar este tipo de procedimiento, siempre que se cuente con la infraestructura y el personal capacitado para realizar cirugías de alta complejidad teniendo como parámetro los resultados aquí publicados.

Estamos totalmente convencidos de que se requiere aumentar el número de casos para que la muestra sea significativa, pero los resultados obtenidos hasta el momento son alentadores.

\section{Agradecimientos}

Al personal médico involucrado en el manejo integral de los pacientes. 


\section{Conflicto de interés}

Los autores declaran no tener conflicto de interés.

\section{Financiamiento}

No se recibió ningún patrocinio para realizar este artículo.

\section{Responsabilidades éticas}

Protección de personas y animales. Los autores declaran que para esta investigación no se han realizado experimentos en seres humanos ni en animales.

Confidencialidad de los datos. Los autores declaran que han seguido los protocolos de su centro de trabajo sobre la publicación de datos de pacientes.

Derecho a la privacidad y consentimiento informado. Los autores han obtenido el consentimiento informado de los pacientes y/o sujetos referidos en el artículo. Este documento obra en poder del autor de correspondencia.

\section{Bibliografia}

1. Acuña-Tovar M, Bassaure EL. Cáncer de páncreas, guías diagnósticas 2013. Hospital General de México. 2013;1:59-66.

2. Ferraro A, Ferraina P. Tumores periampulares. Cirugía Digestiva. 2009;82:1-20.
3. Chapa Azuela O, Roldán García JA, Díaz-Martínez J, Etchegaray-Dondé A. Pancreatoduodenectomía totalmente laparoscópica, primer caso reportado en México. Cir Cir. 2017;85:344-9.

4. Crisanto Campos BA, Arce-Liévano E, Robles-Avila JA. Experiencia inicial en pancreatoduodenectomía laparoscópica en un hospital general de la Cuidad de México. Cirujano General. 2016;38:59-66.

5. Alsfasser G, Hermeneit S. Minimally invasive surgery for pancreatic disease - current status. Dig Surg. 2016;33:276-83.

6. Fernández Cruz L, Cesar-Borges G. Minimally invasive surgery of the pancreas in progress. Langenbecks Arch Surg. 2005;390:342-54.

7. Molina JF, Garnica G. Cirugía laparoscópica de patología pancreática. Cirujano General. 2010;32:25-6.

8. Shimizu S, Tanaka M, Konomi H. Laparoscopic pancreatic surgery. Surg Endosc. 2004;18:402-6.

9. Keck T, Kusters S, Friedrich U. Total laparoscopic pancreatoduodenectomy and reconstruction via laparoscopic pancreatogastrostomy. Langenbecks Arch Surg. 2012;397:1009-12.

10. Date RS, Siriwardena AK. Current status of laparoscopic biliary bypass in the management of non-resectable peri-ampullary cancer. Pancreatology. 2005;5:325-9.

11. Giger U, Michel JM, Wiesli P, Schmid C, Krähenbühl L. Laparoscopic surgery for benign lesions of the pancreas. J Laparoendosc Adv Surg Tech A. 2006;16:452-7.

12. Liu Z, Yu MC, Tan JW. Laparoscopic pancreatoduodenectomy via reverse $V$ approach with four ports: initial experience and perioperative outcomes. World J Gastroenterol. 2015;21:1588-94.

13. Basuch D, Keck T. Laparoscopic pancreatic resections. Langenbecks Arch Surg. 2013:398:939-45.

14. Corcione F, Pirozzi F, Cuccurullo D, Piccolboni D. Laparoscopic pancreatoduodenectomy: experience of 22 cases. Surg Endosc. 2013;27:2131-6.

15. Jacobs MJ, Kamyab A. Total laparoscopic pancreatoduodenectomy. JSLS. 2013;17:188-93.

16. Tran TB, Dua MM, Worhunsky DJ, Poultsides GA, Norton JA, Visser BC. The first decade of laparoscopic pancreatoduodenectomy in the United States: costs and outcomes using nationwide inpatient sample. Surg Endosc. 2016;30:1778-83.

17. Pardo F, Rotellar F, Valenti V, Pastor C. Cirugía laparoscópica hepática y pancreática. An Sist Sanit Navar. 2005;28:51-9.

18. Wang M, Meng L, Cai Y, Li Y, Wang X, Zhang Z, et al. Learning curve for laparoscopic pancreatoduodenectomy: a CUSUM analysis. J Gastrointest Surg. 2016;20:924-35.

19. Abu Hilal M, Hamdan M, Di Fabio F, Pearce NW, Johnson CD. Laparoscopic versus open distal pancreatectomy: a clinical and cost-effectiveness study. Surg Endosc. 2012;26:1670-4.

20. Merkow J, Paniccia A. Laparoscopic pancreatoduodenectomy: a descriptive and comparative review. Chin J Cancer Res. 2015;27:368-75. 